\title{
An ana!ysis of the visual component in recognition memory for verbal stimuli*
}

\author{
KIM KIRSNER† \\ University of Western Australia, Nedlands, Australia 6009
}

\begin{abstract}
Forty Ss were given a continuous recognition memory test in which each word was presented twice, either in the same print or in different print on the two occasions. The results showed that (a) recognition performance was facilitated to a small but statistically significant extent in the same-print condition and that (b) Ss could reliably report first presentation print for recognized items for at least $1 \frac{1}{2} \mathrm{~min}$. In a second experiment, the stimuli used were nonsense strings of from five to seven letters instead of words. This manipulation increased the same-print advantage in recognition but reduced Ss' ability to report first print form. The results indicate that information about the physical features of verbal stimuli is retained in a visual code that is partially or wholly independent of the verbal code for the same stimuli. The results are inconsistent with the conclusion that the visual code is stored only as a dependent attribute of the verbal code in memory.
\end{abstract}

When a person reads a word, one possible assumption is that the item is immediately translated into an auditory-verbal or semantic code. According to this view, the verbal and semantic properties of the word are extracted, whereas normally redundant information about the physical properties of the visual stimulus are lost. However, the results of recent studies (e.g., Kroll, Parks, Parkinson, Beiber, \& Johnson, 1970; Warrington \& Shallice, 1969) show that information about the physical properties of visually presented verbal stimuli may be retained in memory for $10-25 \mathrm{sec}$, a period of time substantially beyond that which could be useful for word identification. The present study was implemented with a view to (a) providing further knowledge about the duration of visual persistence for verbal stimuli and (b) gaining some insight into the coding processes involved in the retention of the representational as distinct from the semantic attributes of verbal stimuli.

The present study used a modification of the continuous trial recognition memory paradigm introduced by Shepard and Teghtsoonian (1961). In their experiment, Ss were exposed to a series of words, within which each item was repeated once, at one of several possible retention intervals. The technique was modified here so that an item could be repeated in the same physical form (e.g., CARROT/CARROT) or in a different physical form (e.g., CARROT/carrot) on the first and second presentations in the continuous series.

Two questions were considered in the present experiment. The first question involved a direct

*This research was supported by Research Grants APA 146 from the National Research Council of Canada and OMHF 164 from the Ontario Mental Health Foundation to B. B. Murdock, $\mathrm{Jr}$. The research was completed while the author was on a postdoctoral fellowship at the University of Toronto. The author wishes to thank B. B. Murdock. F. I. M. Craik, R. Lockart, and E. J. Wells for their assistance in the preparation of this article.

t Requests for reprints should be sent to Kim Kirsner, Department of Psychology. University of Western Australia, Nedlands, Australia 6009. approach in which $S$ was required to report the original presentation form of each item recognized as "old." That is, whenever $S$ recognized that an item had occurred previously in the series, he gave a positive response and indicated whether or not the item was presented in the same form on the two occasions. Mann (1970), in a recall task, found that Ss correctly reported modality of presentation for $98 \%$ of the items recalled, even at a retention interval where representational persistence is not normally considered to be a factor. The present study extended this question to the recognition paradigm where it is possible to sample all presented items, not only those recalled by $S$.

Several explanations could reasonably be advanced to account for form report in the continuous recognition memory paradigm. For example, one possibility is that form report is based on a label or attribute value that is linked with and dependent on the verbal unit in memory. If this is the case and if the recognition decision is based on a comparison between the verbal (or semantic) representations of the memory trace and the probe, then recognition memory should be insensitive to the "visual" similarity of the first and test presentations. That is, recognition performance should be equal in the physically identical (PI) and nominally identical (NI) conditions. On the other hand, if a visual code is considered in the recognition decision, recognition performance should be superior in the PI condition. Finally, by comparing PI and NI at a variety of retention intervals, it should be possible to estimate the duration of representational persistence.

\section{EXPERIMENT I}

\section{Method}

\section{Design}

The experiment was conducted using a mixed design for the 2 by 2 by 2 by 6 factorial combination representing one between-S variable. task (recognition or recognition plus report 


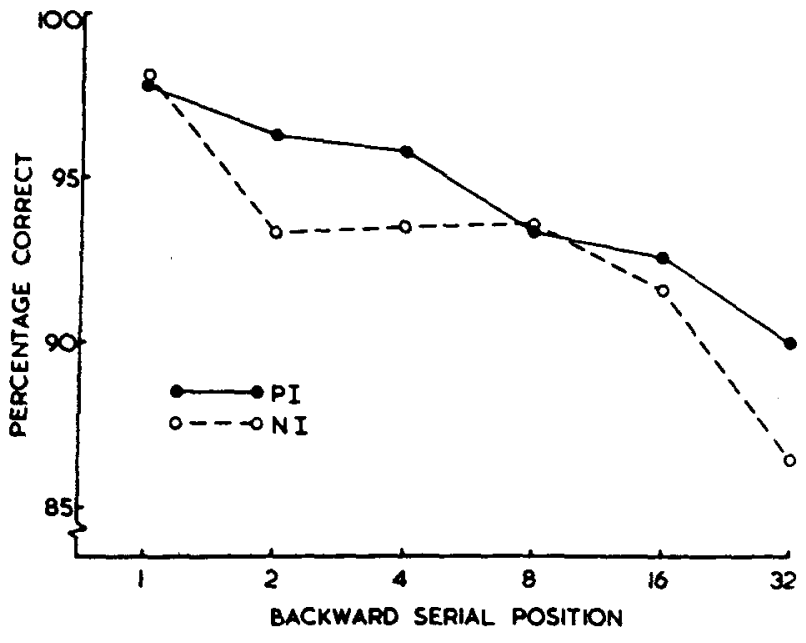

Fig. 1. Experiment I: Percentage correct for words as a function of print combination and recency. $\mathrm{PI}=$ physically identical, $\mathrm{NI}=$ nominally identical.

of original presentation form), and three within-S variables, test form (uppercase or lowercase), similarity (same or different relationship between the original and test presentation forms), and backward serial position $(1,2,4,8,16$, and 32). Backward serial position (BSP) $=1$ refers to an immediate repetition with no interpolated items.

In a single session $S$ s were presented with a continuous series of 532 words. The first 52 words were intended to absorb short-run practice effects and were not considered in the analyses. Within the critical list, each word occurred twice and there were 10 replications of each combination of the experimental conditions. Each replication of the test trials ( 24 combinations) was located within a concealed block of 48 trials. Thus, within each block $\mathbf{P}($ old $)=.5$. The experimental conditions were ordered differently within each replication.

The words, drawn from a pool of common two-syllable nouns of five, six, or seven letters, were presented at a rate of $3 \mathrm{sec}$ per word. Each word was presented for $1.5 \mathrm{sec}$, followed by a blank interval for $1.5 \mathrm{sec}$. Two formats were drawn up, each format constituting a random sample from the word pool. Each format was presented to $10 \mathrm{Ss}$ in each between-S condition.

The items were prepared in the appropriate case on an IBM typewriter. The list was inserted in the typewriter and this was advanced by a solenoid attached to the return button. A Shibadan TV camera was focused on the return carriage and Ss viewed the stimuli on a Shibadan monitor.

\section{Procedure for the Recognition Group}

Ss were required to decide whether or not each word had occurred in the list before. They were instructed to make this decision regardless of whether or not the word was presented in the same form on the two occasions. Ss responded by pressing a "yes" or "no" button on each trial. Ss used the index finger of their dominant and nondominant hands for the yes and no responses, respectively. Reaction time (RT) was measured from the onset of each word.

\section{Procedure for the Recognition Plus Report Group}

Ss were required to say "same," "different," or "no." If a word had occurred in the list before, Ss said same or different, contingent on their decision as to whether or not the word had been presented in the same physical form on the two occasions. Ss said no if the word had not appeared in the list before. RT was not measured in this experiment.

\section{Subjects}

Each group comprised 20 undergraduate $S s$ from the University of Toronto $S$ pool. They were naive with respect to the experiment and were paid for their services on completion of the single session.

\section{Results}

\section{Recognition Accuracy}

The results presented in Fig. 1 show the percentage of correct detections for the PI and NI conditions as functions of BSP. The data are based on 800 observations per point and represent mean correct detections for the $40 \mathrm{Ss}$ from both groups in Experiment I. Figure 1 shows a recency effect for both conditions and a small advantage for the PI condition at four serial positions. An ANOVA (task by Ss by similarity by BSP) showed that the difference between PI and NI was significant $[F(1,38)=9.2, p<.01]$. The means for the PI and NI conditions were $94.3 \%$ and $92.8 \%$, respectively. The effect of BSP was also significant $[F(5,190)=19.1, p<.001]$. The main effect of the task variable $(F<1)$ and the interaction of Task by Similarity $(F<1)$ were not significant. These results, together with the false alarm rates of $9.8 \%$ and $6.5 \%$ in Groups 1 and 2, respectively, show that the presence of the additional form report requirement did not impair performance on the primary recognition task or, indeed, have any effect on the use of representational information in the recognition decision.

\section{Recognition Reaction Time}

The mean RTs for correct detection in the PI and NI conditions were 726 and $738 \mathrm{msec}$, respectively. An ANOVA (Ss by similarity by BSP) showed that the main effects of similarity $[F(1,19)=10.9, p<.01]$ and BSP $[F(5,95)=19.2, p<.001]$ were significant, but the interaction of Similarity by BSP was not significant $[F(5,95)=1.3, p>.05$. The results were based on between 350 and 386 observations per point from the $20 \mathrm{Ss}$ in Group 1. Mean RT for correct rejections was $777 \mathrm{msec}$.

\section{Knowledge of Physical Form}

The results shown in Table 1 represent the proportion of correct form judgments for the same- and different-print conditions in the recognition plus report groups in Experiments I and II. The data depend, of course, on correct recognition. The results show that Ss can retain and use information about the physical form of words almost perfectly if there are no intervening items and with considerable accuracy even after 31 intervening items. There is in fact little or no further change in performance beyond the decrement caused by a single intervening item. Unlike recognition, the data 
show no overall advantage for the same-print condition.

The false alarm data for Experiment I suggest. however, that these results may be contaminated by Ss' biases in giving same and different judgments. For words, 14 out of 20 Ss gave more different responses, and this is reflected in the overall preference shown in the false alarm data in Table 1. A signal detection analysis was implemented in order to overcome this bias. The hit rate for this analysis was calculated from the number of correct form judgments divided by the number of correct recognition detections. False alarm rates were calculated from the allocation of same and different judgments to new items. For this purpose, it was assumed that the population of new items was divided equally into "same" and "different" events. Thus, for a given $S$ the false alarm rate was equal to the number of same judgments divided by half the population of new items. The same procedure was adopted for different judgments. The $\mathrm{d}^{\prime}$ statistic was computed separately for each condition for each $S$.

ANOVAs (Ss by similarity by BSP) were completed on the proportion and signal detection data. The main effect of similarity was not significant in either instance: $F<1$ and $F(1,19)=4.3, p>.05$ in the proportion and signal detection ANOVAs, respectively. The main effect of BSP was significant in both ANOVAs $(p<.001)$ and, although the interaction of Similarity by BSP was significant in the proportion data $[F(5,95)=3.1$, $p<.05]$, this was not the case in the $d^{\prime}$ data $[F(5,95)=1.6, p>.05]$.

\section{DISCUSSION}

The results of Experiment I show, first, that Ss can give reliable reports about original presentation form over a period of at least $90 \mathrm{sec}$ and, second, that recognition memory is facilitated to a small but statistically significant extent in the PI condition. There was some suggestion that this advantage is time or item dependent, but the statistical analyses on the accuracy and RT data support the conclusion that there is an effect relating to PI that is not dissipated at long retention intervals. The results of Experiment $I$ are consistent with two conclusions. First, information about the physical characteristics of verbal stimuli is retained over a period normally associated with long-term as compared to short-term memory, and second, "visual" information is used in the recognition decision.

The notion that the visual information is stored relative to the verbal trace can be reconciled with the recognition results only if it is assumed that attributional as well as verbal information enters the recognition decision. Experiment II was designed to test the hypothesis that information about the visual characteristics of verbal stimuli is stored relative to the verbal trace. If the visual information is dependent on the verbal trace, then it follows that degradation of this
Table 1

Proportion of Correct Physical Form Judgments as a Function of Material, Similarity, and BSP

\begin{tabular}{lllllllll}
\hline & 1 & 2 & 4 & 8 & 16 & 32 & FA \\
\hline Same & 96 & 74 & 73 & 74 & 77 & 75 & 2.3 \\
Different & 95 & 82 & 78 & 77 & 75 & 65 & 3.9 \\
Same & 97 & 74 & 71 & 75 & 70 & 68 & 9.3 \\
Different & 94 & 72 & 49 & 55 & 44 & 54 & 7.9 \\
\hline
\end{tabular}

semantic focus should reduce or eliminate PI facilitation. However, if the visual information is stored independently, in a separate visual store, for example, then PI facilitation should be insensitive to changes in the meaningfulness of the stimuli.

\section{EXPERIMENT II}

The design and procedure for Experiment II followed that of Experiment I, except that the letters for each word were scrambled according to one of a set of numerical sequences for each word length. For example, CARROT became RCTORA. No attempt was made to control either pronounceability or the number of syllables in each string and the items varied considerably in these respects. The word lists were those used in Experiment I. Each word was scrambled according to the same sequence on both presentations.

\section{Results}

\section{Recognition Accuracy}

As shown in Fig. 2, the scrambling manipulation has clearly reduced the level of performance. For example, percent correct in the NI condition at BSP 32 has declined from 85 in Experiment I to 37 in Experiment II. But, in comparison with a false alarm rate of $18 \%$, this performance level is still better than chance. Performance in the PI condition is clearly superior to NI at all sampled BSPs, and there is no evidence of a decline in the PI advantage at long retention intervals. If anything, the PI advantage increases with increasing BSP. An ANOVA (task by Ss by similarity by BSP) showed that the difference between PI and NI $[F(1,38)=127.1, p<.001]$ and the interaction of Similarity by BSP $[F(5,190)=4.5$. $p<.001]$ were both significant. As in Experiment I, the task factor $[F(1,38)=2.9, p>.05]$ was not significant and, apart from BSP $[F(5,190)=122.0, p<.001]$. there were no other significant effects.

\section{Recognition Reaction Time}

The mean RTs for correct detections in the $\mathrm{PI}$ and NI conditions were 890 and $924 \mathrm{msec}$. respectively. There was a clear advantage for the PI condition at BSPs 1. 2. 


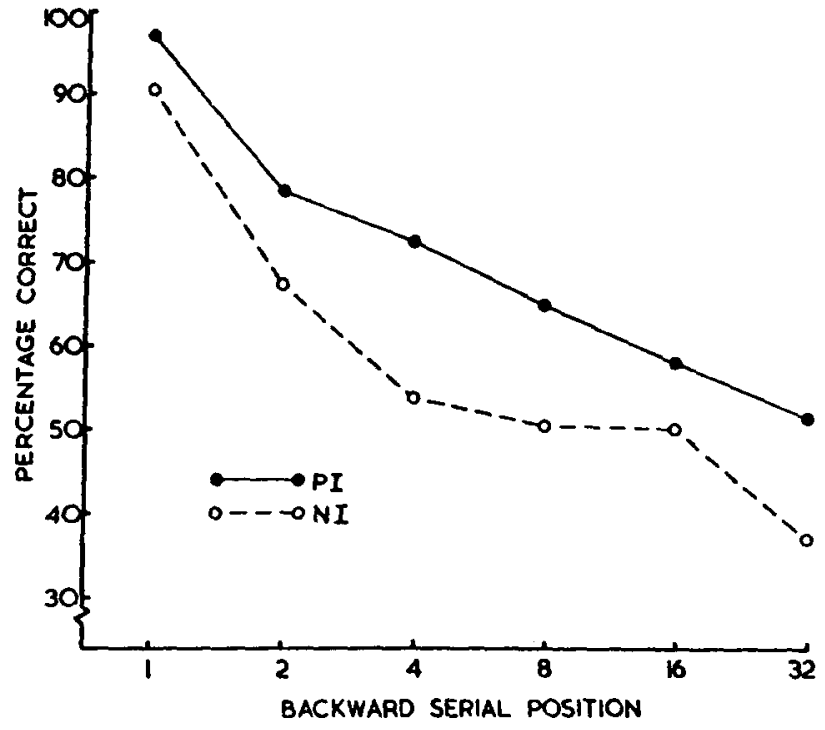

Fig. 2. Experiment II: Percentage correct for nonsense strings as a function of print combination and recency.

and 4 , but no difference was found between the PI and NI conditions for the combined data from BSPs 8,16 , and 32. The data from these BSPs were pooled owing to the high error rate. The pooling criterion, chosen in advance, was that each mean should represent at least three values per $S$ for the $20 \mathrm{Ss}$ in the recognition group. An ANOVA showed that the main effects of similarity $[F(1,19)=22.8, p<.001]$ and BSP $[F(1,19)=22.0$, $\mathrm{p}<.001]$ were significant. The interaction of Similarity by BSP was also significant $[F(3,57)=3.6, p<.05]$. The mean RT for correct rejections was 904 msec.

\section{Knowledge of Physical Form}

The proportion data presented in Table 1 show that performance on the judgment task is considerably reduced in Experiment II, where nonsense items were used as stimuli. There is, however, a clear advantage for the same-print condition, particularly at long retention intervals, although this may be contaminated by response bias factors which, in Experiment II, favored the "same" judgment. The data analyses followed the procedure outlined for Experiment $\mathbf{I}$. There was a significant advantage for the same-print condition in the proportion analysis $[F(1,19)=6.6, p<.05]$ but this was eliminated in the $\mathrm{d}^{\prime}$ analysis $[\mathrm{F}(1,19)=2.9$, $p>.05]$. The interaction of Similarity by BSP was also affected by the transformation, from $F(5,95)=3.5$, $\mathrm{p}<.01$ in the proportion analysis to $F(5,95)=2.3$, $\mathrm{p}<.05$ in the $\mathrm{d}^{\prime}$ analysis.

A further ANOVA (material by Ss by similarity by BSP) was completed to test the difference between the word and nonsense experiments. The main effect of material was significant $(p<.001)$ in the proportion and $\mathrm{d}^{\prime}$ analysis.

\section{DISCUSSION}

The recognition results are inconsistent with both an independent and a dependent model of the relationship between the verbal and visual codes in memory. If an independent visual code was retained for each verbal stimulus, performance in the PI condition should be insensitive to the semantic manipulation. This was not the case. It is possible, however, to reconcile the independent model with the recognition results in the second experiment by assuming that, while the visual memory code is stored independently, the final decision mechanism operates on input from both the verbal and the visual codes. On the other hand, semantic degradation was associated with an increase in the magnitude of PI facilitation, indicating that the visual code can be used in the recognition decision in the absence of a clear verbal or semantic focus. Thus, the recognition results are also inconsistent with a model in which representational information is stored only as an abstract attribute or label which is dependent on and accessed through the verbal unit.

While it is now well established that Ss can provide reliable reports about a number of the attributes of verbal stimuli (Bray \& Batchelder, 1972; Hintzman, Block, \& Inskeep, 1972), the coding basis of this skill remains obscure. Undoubtedly the verbal trace is critical in attribute report-indeed the notion of attribute retention becomes somewhat meaningless in the absence of a common verbal unit-but there is evidence that attribute retention is sensitive to perceptual factors as well as to the semantic factors shown in the present results. For example, experiments by Hintzman et al (1972) and Kirsner (in preparation) have shown that retention of the modality and within-modality attributes is not equal. Given equal perceptual discriminability among the attribute dimensions concerned, their findings support the view that attribute recall is based on perceptual as well as verbal information. Further support for this position comes from the finding (Madigan \& Doherty, 1971) that, while a verbal associate (digit) has a high cost on item recall, an attribute associate (location) does not.

The results of the present experiments raise two further problems for recent descriptions of visual persistence. First, with regard to the capacity of visual memory, they show that information can be retained about 30 or more five- to seven-letter strings. This stands in contrast to the results of a recall experiment by Parkinson (1972), who concluded that little or no visual information is retained when the presentation set includes more than three letters. Second, with regard to visual rehearsal or "visualization," a mechanism that has been used to explain short-term visual persistence (e.g., Parks, Kroll, Parkinson, \& Salzberg, 1972; Posner, Boies, Eichelman, \& Taylor, 1969), it does not seem likely that PI facilitation in the present study is due to an active visual maintenance strategy. While these differences 
appear to suggest that the technique used here samples information from yet another visual memory system, this need not be the case. An alternative and more parsimonious explanation is that, apart from iconic memory, there is a single long-term visual memory system for verbal stimuli. In this account it is necessary to assume that the contribution made by the visual memory system to performance in a given experiment is contingent on the nature of the task, the stimulus material, and temporal parameters. For example, the capacity and temporal distinctions between the present results and those of Parkinson (1972) could reflect differences in the amount of visual information necessary to influence performance in recognition as compared to recall. In any case, in the absence of qualitative evidence such as that advanced by Doost and Turvey (1971) to separate iconic memory, additional process distinctions within the domain of visual memory may not be justified.

To summarize, the results are consistent with a description of visual memory for verbal stimuli in which (1) a visual code is retained either partially or wholly independent of the verbal unit and (2) the verbal and visual memory codes retaining information about the occurrence of verbal stimuli are closely linked. The results are inconsistent with a description in which representational information is stored only as an abstract attribute, dependent on retention of the verbal unit in memory.

\section{REFERENCES}

Bray, N. W., \& Batchelder, W. H. Effects of instructions and retention interval on memory for presentation mode. Journal of Verbal Learning \& Verbal Behavior, 1972, 11, 367-374.

Doost, R, \& Turvey, M. T. Iconic memory and central processing capacity. Perception \& Psychophysics, 1971, 9 , 269-274.

Hintzman, D. I., Block, R. A., \& Inskeep, N. R. Memory for mode of input. Journal of Verbal Learning \& Verbal Behavior, 1972, 11, 741-749.

Kroll, N. E. A., Parks, T., Parkinson, S. R., Beiber, S. L., \& Johnson, A. L. Short-term memory while shadowing: Recall of visually and aurally presented letters. Journal of Experimental Psychology, 1970, 85, 220-224.

Madigan, S., \& Doherty, L. Retention of item attributes in free recall. Psychonomic Science, 1972, 27, 233-235.

Mann, J. E. Modality effects in free recall ordered by presentation mode. Unpublished bachelor's thesis, University of Toronto, 1970.

Parkinson, S. R. Short-term memory while shadowing: Multiple-item recall of visually and of aurally presented letters. Journal of Experimental Psychology, 1972, 92, 256-265.

Parks, T. E., Kroll, N. E. A., Parkinson, S. R., \& Salzberg, P. M. The varieties of visual memory. American Scientist, in press.

Posner, M. I., Boies, S. J., Eichelman, W. H., \& Taylor, R. L. Retention of visual and name codes of single letters. Journal of Experimental Psychology Monograph, 1969, 79, 1-16.

Shepard, R. N., \& Teghtsoonian, M. Retention of information under conditions approaching a steady state. Journal of Experimental Psychology, 1961, 62, 302-309.

Warrington, E. K., \& Shallice, T. The selective impairment of auditory verbal short-term memory. Brain, 1969, 92, 885-896.

(Received for publication January 31, 1973; revision received April 5, 1973.) 